\title{
DYNAMIC GROWTH OF ANTI-PLANE SHEAR CRACKS IN IDEALLY PLASTIC CRYSTALS
}

\author{
Ruzica R. NIKOLIC and James R. RICE \\ Division of Applied Sciences, Harvard University, Cambridge. MA O2138. U.S.A.
}

Received 18 July 1988

\begin{abstract}
A near-tip asymptotic analysis is given for the stress and deformation field near the tip of crack propagating dynamically under anti-plane shear in an idealiy plastic single crystal. A particular class of orientations of the crack relative to the crystal is considered so that the yield locus is of simple diamond shape (relative to directions along and perpendicular to the crack) in the plane of the anti-plane shear stresses. The near-tip solution is shown to consist of sectors which carry constant stresses, at yield levels, corresponding to adjacent vertices on the diamond-shaped yield locus, and which are joined along an elastic-plastic shock discontinuity. All plastic flow in the near-tip region occurs in the shock. Plastic strains and particle velocity are finite at the crack tip. The plastic strain is proportional to the elastic strain at onset of yielding and is inversely proportional to the elastic Mach number associated with the speed of crack growth.
\end{abstract}

\section{Introduction}

Dynamic crack growth in ideally plastic single crystals is analyzed here for geometries and orientations such that two-dimensional states of antiplane shear constitute a possible deformation field. The analysis is asymptotic; the limit $r \rightarrow 0$ is considered where $r$ is distance from the moving crack tip. Cases of stationary and quasistatically growing anti-plane cracks for different orientations in f.c.c. and b.c.c. crystals were solved by Rice and Nikolic (1985). Here inertia effects are taken into account for the growing crack. The material yields according to the attainment of a critical value for the resolved shear stress on one or more different slip systems in a crystal. Since for a perfectly plastic material the shear wave velocity for an appropriate direction of straining is zero, the crack growth is supersonic even at small speeds. Thus, the inertia terms in the basic equations may have a significant effect on the nature of near tip fields. It may also be expected that the quasistatic solution includes features that will not be present in dynamic results. For Mode III crack growth in isotropic ideally plastic solids, this was shown by Slepyan (1976) and confirmed by Achenbach and Dunayevsky (1981), and Freund an Douglas (1982). These authors discovered that the Mode III dynamic solution. unlike the quasistatic one for a growing crack, predicts no elastic unloading sectors and the entire field around the crack tip is plastic. The shear strain has a logarithmic singularity. The solutions considered here are different because we consider the behavior of single crystals, not the isotropic material.

For the stationary crack cases in crystals the platic zone at a crack tip collapses into discrete planes of displacement and stress discontinuity emanating from the tip. For the quasistatically growing crack these same planes also constitute collapsed plastic zones in which velocity and plastic strain discontinuities occur but across which the stresses and anti-plane displacement are fully continuous. For the dynamic growth case considered in the present work the configuration of the stress field around the crack tip is expected to be quite different. We considered different types of near-tip solutions to the equations governing dynamic growth of a crack in anti-plane shear, like elastic and plastic sectors. both of constant and variable stresses. We conclude that the whole near-tip field around the crack tip is plastic (or is at least stressed to a level meeting the yield condition). For the range of the coordinate angle $\theta$ of 0 to $180^{\circ}$ the solution consists of two plastic sectors of constant stresses with the houndary between 


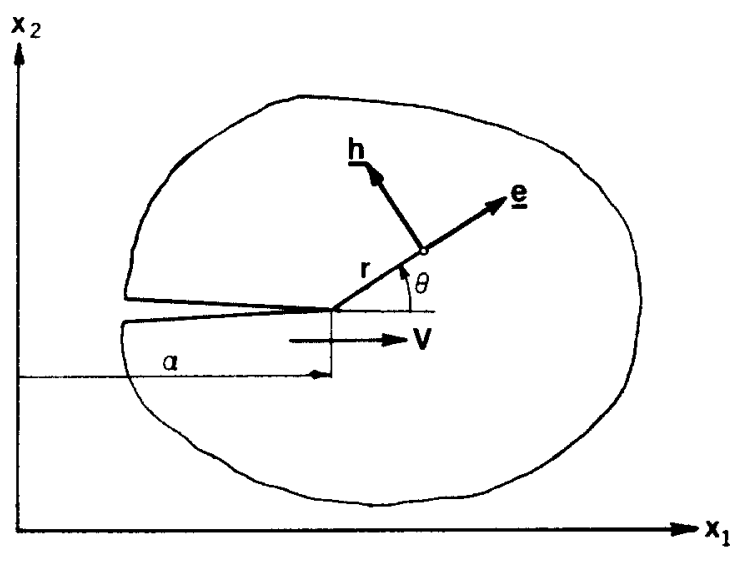

(a)

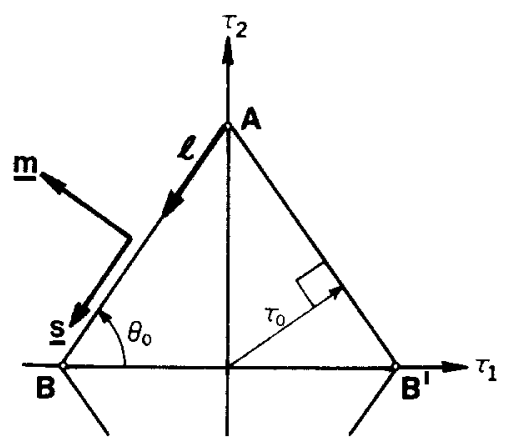

(b)

Fig. 1. (a) Coordinate systems and notation used: $x_{1} x_{2}$ Cartesian coordinate system; $r, \theta$ polar coordinates centered at the crack tip; $\boldsymbol{e}$ and $\boldsymbol{h}$ are unit vectors in the radial and angular directions; $a$ is crack length; $V$ is crack propagation speed, $\mathrm{d} a / \mathrm{d} t$. (b) Upper part of the yield surface; $l$ is arc length along the active yield surface segment; $\boldsymbol{m}$ is the unit vector normal to the active segment and $s$ is a unit vector along it.

them being an elastic-plastic shock wave across which stress and particle velocity are discontinuous, and along which a finite plastic strain is generated. There is no strain singularity, so long as crack speed and material density are regarded as non-zero. All other types of combination of sectors with different local solution-types could not constitute the solution because they did not satisfy either necessary continuity conditions between sectors, or boundary conditions at the crack surface, or both.

Coordinate systems used throughout the paper as well as the yield surface, for the particular class of crack orientations and single crystal considered, are shown in Fig. 1. For example, the yield surface geometry may represent a (100) crack propagating in the [011] direction in a face-centered or body-centered cubic metal crystal, and also some other cases of interest (see Rice and Nikolic, 1985). The fixed Cartesian coordinate system $x_{1} x_{2} x_{3}$ is chosen so that the $x_{3}$ axis is parallel to the crack front and $x_{1}$ points in the direction of crack growth. Polar coordinates $r, \theta$ have their origin at the moving crack tip, lie in the $x_{1} x_{2}$ plane, and have associated unit vectors $e$ and $\boldsymbol{h}$ in the radial and angular directions, respectively. It is evident, from Fig. 1, that

$\partial r / \partial x_{\alpha}=e_{\alpha}, \quad \partial \theta / \partial x_{\alpha}=h_{\alpha} / r$,

where

$e_{1}=h_{2}=\cos \theta, \quad e_{2}=-h_{1}=\sin \theta$,

$e_{3}=h_{3}=0$.

Greek indices have values 1,2 and follow the summation convention. Exception is made for $\theta$; indices $r$ and $\theta$ always denote components relative to the polar coordinates. The crystal orientations and loadings considered here are such that the only existing stress components are $\sigma_{13}=\sigma_{31}$ $\equiv \tau_{1}$ and $\sigma_{23}=\sigma_{32} \equiv \tau_{2}$.

\section{Governing equations and general assumptions for fields around the crack tip in limit $r \rightarrow 0$}

The equation of motion for anti-plane shear in Cartesian coordinates is

$\tau_{\alpha, \alpha}+f=\rho \dot{v}$ 
where $f$ is the body force, $\rho$ is the material density, $v$ is the particle velocity and $\dot{v}$ is the acceleration. In polar coordinates

$$
\left(\partial \tau_{\alpha} / \partial \theta\right) h_{\alpha}+r\left(\partial \tau_{\alpha} / \partial r\right)+r f=r \rho \dot{v} .
$$

For the asymptotic analysis of the stress field around the crack tip it is assumed that, because of boundedness of stress, $r\left(\partial r_{\alpha} / \partial r\right) \rightarrow 0$ as $r \rightarrow 0$, so the second term in (2.2) vanishes. Also, since the dominant part of $\dot{v}$ is $-V_{v_{1}}$ near the moving tip,

$r \dot{v} \rightarrow-V r v, 1$

as $r \rightarrow 0$ where $V$ is the crack propagation speed.

Thus the equation of motion requires

$h_{a \mathrm{r}} \tau_{a}^{\prime}=-\rho V r v,_{1}$

as $r \rightarrow 0$ where $\tau_{\alpha}^{\prime}=\lim _{r \rightarrow 0}\left[\partial \tau_{\alpha}(r, \theta, t) / \partial \theta\right]$. Note also that

$\lim _{r \rightarrow 0}\left(r \dot{\tau}_{c r}\right)=\lim _{r \rightarrow 0}\left[r\left(\partial \tau_{\alpha} / \partial \theta\right) \dot{\theta}\right]=e_{2} V \tau_{\alpha x}^{\prime}$

since $\dot{\theta}=e_{2} V / r$.

The plastic yield condition is in general written as $f(\boldsymbol{\sigma})=0$ where $\boldsymbol{\sigma}$ is the stress tensor, and in the two-dimensional stress space with coordinates $\tau_{\alpha}$ this represents an equation of a curve. For single crystals this curve is a polygon, with flat segments because slip can occur only on certain planes and in certain directions, i.e., on particular slip systems. The yield condition for crystals is thus written as

$$
\begin{aligned}
\tau^{(k)} & \equiv n_{i}^{(k)} \sigma_{i j} s_{j}^{(k)}=n_{3}^{(k)} \tau_{\alpha \alpha} s_{\alpha}^{(k)}+n_{\alpha}^{(k)} \tau_{\alpha} s_{3}^{(k)} \\
& \leqslant \tau_{0}^{(k)}(i, j=1,2,3 ; \alpha=1,2)
\end{aligned}
$$

where $\tau^{(k)}$ is the resolved shear stress on the $k$-th slip system, $n_{3}^{(k)}, n_{\alpha}^{(k)}$ are the components of the unit normal of the slip plane, $s_{3}^{(k)}, s_{\alpha}^{(k)}$ are the components of the unit vector in the slip direction, and $\tau_{0}^{(k)}$ is the yield strength of the $k$-th slip system. Thus, for each slip system of a crystal, in the two-dimensional $\tau$ plane we obtain a line given by the equation $m_{\alpha}^{(k)} \tau_{\alpha}=\tau_{0}^{(k)}$, where $\boldsymbol{m}^{(k)}=$ $\left(m_{1}^{(k)}, m_{2}^{(k)}\right)$ is a vector in the outer normal direction to the given line. (Later $\boldsymbol{m}$ is regarded as a unit vector, although it need not be so by this definition, and $\tau_{0}$ as the critical shear stress in the $x_{1}, x_{2}$ plane, as marked in Fig. 1(b). E.g., $m_{1}=$ $-\sin \theta_{0}$ and $m_{2}=\cos \theta_{0}$ for flat $A B$ in Fig. 1(b).) The yield surface of a crystal is the inner envelope of the set of these lines.

The constitutive equation. The rate of deformation is given by

$\dot{\gamma}_{\alpha} \equiv v,_{\alpha}=\dot{\gamma}_{\alpha}^{c}+\dot{\gamma}_{k}^{\mathrm{n}}$.

The elastic deformation is given by

$\gamma_{\alpha}^{\mathrm{e}}=c_{\alpha \beta} \tau_{\beta}$

where $c_{\alpha \beta}=c_{\beta \alpha}$ is the $2 \times 2$ matrix of elastic compliances and the plastic strain rate is given by

$\dot{\gamma}_{a}^{\mathrm{p}}=\sum_{k} \dot{\gamma}^{(k)}\left(n_{3}^{(k)} s_{(k}^{(k)}+n_{(k)}^{(k)} s_{3}^{(k)}\right)=\sum_{k} \dot{\gamma}^{(k)} m_{n}^{(k)}$.

where $\dot{\gamma}^{(k)} \geqslant 0$, and the summation is done over all active slip systems, with index $k: \dot{\gamma}^{(k)}>0$ is possible only if equality holds in (2.6). For elastically isotropic crystals (2.8) can be written as

$\gamma_{i r}^{e}=(1 / \mu) \tau_{i r}$

where $\mu$ is the shear modulus. This also holds for some high symmetry orientations of cubic crystals. Sometimes, several symmetrically oriented slip systems must be active simultaneously to produce anti-plane strain only. Thus, a single yield surface segment can correspond to two or more active slip systems. The flow rule is of associated type and this corresponds to normality of $\dot{\gamma}_{i r}^{p}$ to the yield surface in the $\tau_{\alpha}$ plane or, at a vertex, to $\dot{\gamma}_{n}^{p}$ having the direction within the fan defined by limiting outer normals. The constitutive equation (2.7)-(2.9) thus leads, as $r \rightarrow 0$, to

$r v,_{\alpha}=e_{2} V c_{\alpha \beta} \tau_{\beta}^{\prime}+\Lambda m_{\alpha}\left[+\tilde{\Lambda} \tilde{m}_{\alpha}\right]$

where (2.5) has been taken into account. Here $\Lambda \geqslant 0$ and $\tilde{\Lambda} \geqslant 0$ are scalars, $m_{\alpha}$ and $\tilde{m}_{\alpha}$ are the components of the normals to the yield surface segments, and the expression including the term in square brackets is for the state at a vertex where then $m_{\alpha}$ and $\tilde{m}_{\alpha}$ are the limiting normals of the fan. Here $A=0$ if $m_{\alpha} \tau_{\alpha}<\tau_{0}$ (i.e.. inside the yield surface); $1 \geqslant 0$ if $m_{x x} \tau_{x x}=\tau_{0}$ (on the yield surface). 
and $m_{\alpha} \tau_{\alpha}>0$ is impossible. Similar relations also hold for $\tilde{\Lambda}$.

Note here that for elastic deformation, or for the yielding along a single flat segment of the yield surface $r v,{ }_{\alpha}$ is finite as $r \rightarrow 0$ if $\tau_{\beta}^{\prime}$ is finite. This is evident from (2.11) for an elastic sector. and follows from (2.4) and (2.11) for yield along a flat, after the latter is multiplied by $s_{\alpha}$ (Fig. 1(b)). For finiteness of $r v,_{\alpha}$ it is necessary that material velocity is of the form

$v_{\alpha}=-A \ln r+f(\theta)$

where $f(\theta)$ is any differentiable function of the coordinate angle, and $A$ is independent of $\theta$. Further, if the $\tau_{\beta}^{\prime}=0$, then $A=0$ and $f(\theta)$ is constant.

In the next section we show that all relevant equations are satisfied if the state near the crack tip, over $0<\theta<180^{\circ}$, consists of two angular sectors that are stressed to yield levels and that are separated by an elastic-plastic shock wave. There is a stress and velocity jump across the shock, and the analysis which we give of it is equivalent to using (2.4) and (2.11) with $\tau_{\alpha}^{\prime}$ and $r v_{\alpha}$ allowed to have Dirac singularities at the shock angle. Full details of the solution are given in subsection 3.1.

Other types of continuous solutions to (2.4) and (2.11), valid within particular angular sectors, are presented in Subsections 3.2-3.4. However, as explained in Section 4, it does not seem to be possible to assemble such sectors to provide an acceptable near-tip solution other than that with the shock, as developed in Subsection 3.1.

\section{Types of solutions for near tip stress and strain fields}

Around the crack tip two types of zones can be present: zones of material that currently respond elastically (either they had previously yielded but now respond elastically, or had always responded only elastically), and zones of material that are stressed to yield levels and may currently respond plastically. The full stress and strain field around the tip of a dynamically growing crack could be a combination of these zones. In the following sub- sections we consider different types of sectors that may arise around the tip, and later show which of these and under what conditions can be assembled to form the full field around the tip of the dynamically growing crack. First we discuss the conditions for the existence of a strong shock discontinuity and where in the full field it may appear. and show that all conditions of the problem are satisfied by a near-tip field with such a shock discontinuity.

\subsection{Shock analysis}

Here we examine the conditions for a surface of strong dicontinuity, i.e., a shock, to be a boundary between two sections in the full field around a crack tip. The surface of strong discontinuity is a surface across which components of stress, strain or material velocity jump. In the analysis of quasistatically moving discontinuities by Drugan and Rice (1984), variations of stress and strain across the discontinuity surface are required to obey the same governing equations and constitutive assumptions as they satisfy outside the surface. Drugan and Shen (1987) have adopted the same requirement in a dynamic analysis and have proven that, in general, for anti-plane shear the discontinuity surface has to move at the elastic wave speed for stress changes to accumulate across it. An exeption occurs when the yield surface contains a flat segement, as for the crystals considered here, and then an elastic-plastic shock, moving at a lower wave speed, is possible.

Let us consider a discontinuity surface $S$ that moves at a speed $c$, Figure 2(a). The momentum conservation equation requires that

$\left(\tau_{\alpha}-\tau_{\alpha}^{+}\right) h_{\alpha}=\rho V e_{2}\left(v-v^{+}\right)$.

where the fact that $c=V e_{2}=V \sin \theta$ has been used. Note here that superscript + denotes the value of the quantity in front of the discontinuity surface $S$, and - the value behind it. Quantities without superscripts, like the $\tau_{\alpha}$ and $v$ in (3.1), denote general values along the succession of states traversed at the discontinuity; e.g., the discontinuity follows a path in the $\tau_{1}, \tau_{2}$ plane of Fig. 1(b). 


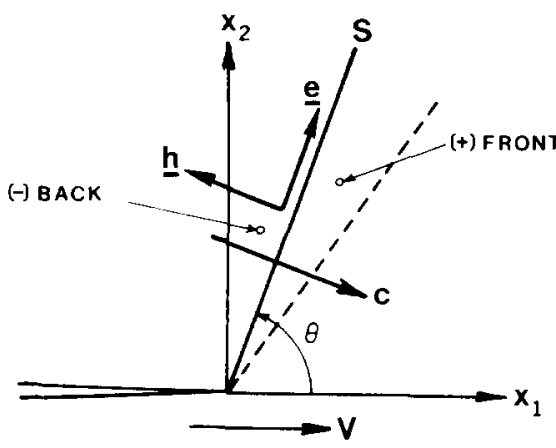

(a)

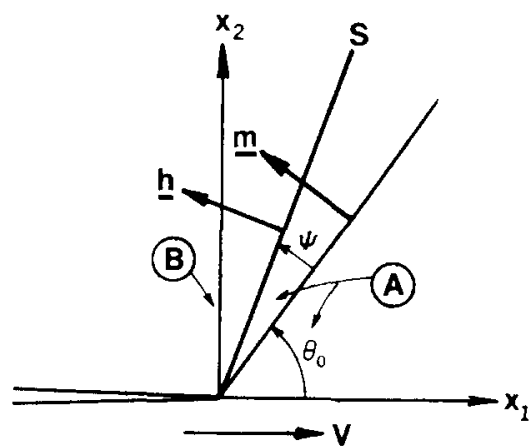

(b)

Fig. 2 (a) The discontinuity surface $S: c$ is the speed of the discontinuity surface normal to itself. (b) The orientation of the discontinuity line $S$ with respect to $\theta_{0}$, the angle of the yield surface in Fig. 1(b): $\psi$ is the angle that defines the orientation of the shock.

We view the discontinuity as the limit of a continuously variable transition with $\theta$. From the continuity of anti-plane displacement $u$ at the moving shock, we can write

$\mathrm{d} \llbracket u \rrbracket / \mathrm{d} t=\llbracket \partial u / \partial t \rrbracket+\llbracket \partial u / \partial x_{\alpha} \rrbracket\left(\mathrm{d} x_{\alpha} / \mathrm{d} t\right)=0$.

Note here that double square brackets denote the jump in a quantity at $S$. The substitutions $\partial u / \partial t$ $=v, \partial u / \partial x_{\alpha}=\gamma_{\alpha}$, and $\mathrm{d} x_{\alpha} / \mathrm{d} t=c_{\alpha}=-c h_{\alpha}$ give

$v-v^{+}=V e_{2} h_{\alpha}\left(\gamma_{\alpha}-\gamma_{\alpha}^{+}\right)$.

Also, from continuity of $u$, and hence $\partial u / \partial r$,

$e_{\alpha}\left(\gamma_{\alpha}-\gamma_{\alpha}^{+}\right)=0$.

From (3.3), with use of (3.4), we can solve for the jump in strain as

$$
V e_{2}\left(\gamma_{\alpha}-\gamma_{\alpha}^{+}\right)=h_{\alpha}\left(v-v^{+}\right)
$$

and then using this in (3.1) we can get

$\left(\tau_{\alpha}-\tau_{a}^{+}\right) h_{\alpha} h_{\beta}=\rho V^{2} e_{2}^{2}\left(\gamma_{\beta}-\gamma_{\beta}^{+}\right)$.

Equation (3.6) shows that in order for strain to accumulate at the shock the stress must change also (unless $\theta=0$ or $\pi$ so that $e_{2}=0$ ). Thus no finite contribution to the jump can come from a vertex state since the stress cannot change there. It must all come from a flat segment of the yield surface; Fig. 1(b). We assume that the + state corresponds to the vertex state $A$ with $\tau_{\alpha \alpha}{ }^{{ }}=\tau_{\alpha}{ }^{4}$ and $l^{+}=0 ; l$ is the arc length along the active yield surface segment and thus has units of stress. From Fig. 1(b),

$\tau_{\alpha}-\tau_{\alpha}^{+}=l s_{\alpha}$,

where $s_{\alpha}$ is the unit vector along the flat. From the constitutive equation, we can then write

$\gamma_{\alpha}-\gamma_{\alpha}^{+}=c_{\alpha \beta} s_{\beta} l+\lambda m_{\alpha}$,

where $\lambda \geqslant 0$ is a scalar giving the plastic strain accumulated, and which is necessarily non-decreasing along the path of traversal of the discontinuity (for non-negative plastic work) $\lambda^{+}=0$ at $A$. Now from (3.6) and (3.7) we have

$h_{\alpha} s_{\alpha} l h_{\beta}=\rho V^{2} e_{2}^{2}\left(\gamma_{\beta}-\gamma_{\beta}^{+}\right)$

and this used in (3.8) gives

$h_{\alpha} s_{\alpha} l h_{\beta}=\rho V^{2} e_{2}^{2}\left(c_{\beta \gamma} s_{\gamma} l+\lambda m_{\beta}\right)$.

Multiplying (3.10) with $s_{\beta}$ (for $l \neq 0$ ) one obtains

$\left(h_{\alpha} s_{\alpha}\right)^{2}=\rho V^{2} e_{2}^{2} s_{\alpha} c_{\alpha \beta} s_{\beta}$

which is the condition that determines the shock orientation. Here we have two solutions, i.e.,

$h_{\alpha} s_{\alpha}= \pm V e_{2} \sqrt{\rho s_{\alpha} c_{\alpha \beta} s_{\beta}}$. 
Multiplying (3.10) with $e_{\beta}$, and taking into account that $e_{\beta} m_{\beta}=h_{\beta} s_{\beta}$, we can solve for $\lambda$ as

$\lambda=l\left[\left(h_{\lambda} m_{\lambda} / h_{\mu} s_{\mu}\right) s_{\alpha} c_{\alpha \beta} s_{\beta}-m_{\alpha} c_{\alpha \beta} s_{\beta}\right]$.

Since we require that $\mathrm{d} l / \mathrm{d} l \geqslant 0$ it follows that the negative solution for $h_{\alpha} s_{\alpha}$ in (3.12) has to be rejected, and that the shock can form only at the positive value of $h_{\alpha} s_{\alpha}$ given in (3.12), Fig. 2(b). From the geometry of the problem

$h_{\alpha} s_{\alpha}=\sin \psi ; \quad e_{2}=\sin \left(\theta_{0}+\psi\right)$

where $\theta_{0}$ (the same angle as for the yield locus in Fig. 1(b)) is the orientation of the discontinuity in the quasistatically growing crack case (Rice and Nikolic, 1985) and $\psi$ is the difference from that orientation to the orientation of the shock. Thus, with the use of (3.14) the orientation of the shock wave can be obtained from (3.12) as

$\sin \psi / \sin \left(\theta_{0}+\psi\right)=V \sqrt{\rho s_{\alpha} c_{\alpha \beta} s_{\beta}}$,

and the total plastic strain accumulated in the shock is thus $\lambda^{-} m_{\alpha}$, where

$\lambda^{-}=l^{-}\left[(\cos \psi / \sin \psi) c_{s s}-c_{m s}\right]$,

where $c_{s s}=s_{\alpha} c_{\alpha \beta} s_{\beta}$ and $c_{m . s}=m_{\alpha} c_{\alpha \beta} s_{\beta}$. (Here the notations $c_{s s}$ and $c_{m s}$ are used since these are components of the compliance matrix associated with the " $m$ " and " $s$ " directions.)

If we introduce a "Mach number" type of notation, $M=V \sqrt{\rho c_{s s}}$, we can solve for $\tan \psi$ from (3.15) as

$\tan \psi=M \sin \theta_{0} /\left(1-M \cos \theta_{0}\right)$,

which used in (3.16) gives

$\lambda^{-}=l^{-}\left\{\left[\left(1-M \cos \theta_{0}\right) / M \sin \theta_{0}\right] c_{s s}-c_{m s}\right\}$.

For the elastically isotropic material $M=$ $V / \sqrt{\mu / \rho}$ is a Mach number, and (3.18) in that case is

$\lambda^{-}=\left(l^{-} / \mu\right)\left[\left(1-M \cos \theta_{0}\right) / M \sin \theta_{0}\right]$.

Note that if the jump is from vertex $A$ to vertex $B$ in Fig. 1(b), then the state reached after the shock has $\tau_{2}=0$ and thus meets the boundary condition on the crack face. In that case

$$
\begin{aligned}
l^{-} & =(\overline{A B})=\left[\left(\tau_{0} / \sin \theta_{0}\right)^{2}+\left(\tau_{0} / \cos \theta_{0}\right)^{2}\right]^{1 / 2} \\
& =\tau_{0} / \sin \theta_{0} \cos \theta_{0},
\end{aligned}
$$

which used in (3.19), for the elastically isotropic case, gives the total plastic strain accumulated in the shock as

$$
\begin{aligned}
& \left(\gamma_{\alpha}^{p}\right)^{-}-\left(\gamma_{\alpha}^{p}\right)^{+} \\
& \quad=m_{\alpha}\left(\tau_{0} / \mu\right)\left[\left(1-M \cos \theta_{0}\right) / M \sin ^{2} \theta_{0} \cos \theta_{0}\right]
\end{aligned}
$$

and a velocity jump, from (3.3) and (3.8) also, of $v^{--}-v^{+}=\sqrt{\mu / \rho}\left(\tau_{0} / \mu \sin \theta_{0} \cos \theta_{0}\right)$.

Analogous expressions are easily written for the general elastically anisotropic case, based on (3.18) rather than (3.19).

Thus we observe that a valid near tip solution is found with the following characteristics: Over $0<$ $\theta<\theta_{0}+\psi$, the stress is constant at the state of vertex $A$, Fig. 1(b),

$\tau_{1}=0, \quad \tau_{2}=\tau_{0} / \cos \theta_{0}$,

with $v=v^{+}=0$ and $\gamma_{\alpha}^{\mathrm{p}}=\left(\gamma_{\alpha}^{\mathrm{p}}\right)^{+}=0$, i.e., the sector is non-deforming. Over $\theta_{0}+\psi<\theta \leqslant \pi$ the stress is again constant but at the state of vertex $B$,

$\tau_{1}=\tau_{0} / \sin \theta_{0}, \quad \tau_{2}=0$

with $v=$ constant $=v^{-}$and $\gamma_{\alpha}^{\mathrm{p}}=$ constant $=\left(\gamma_{\alpha}^{\mathrm{p}}\right)^{-}$. so that this sector is again non-deforming. Here $\left(\gamma_{\alpha}^{\mathrm{P}}\right)^{-}$and $v^{-}$are given by (3.21) and (3.22) above, or their elastically anisotropic generalizations, with $\left(\gamma_{\alpha}^{\mathrm{p}}\right)^{+}=v^{+}=0$. All the plastic deformation thus occurs in the elastic-plastic shock at $\theta=\theta_{0}+\psi$, and we observe that both the plastic strain and particle velocity are finite at the tip. While finite, the strain of this solution varies as $1 / M$, i.e., as $\sqrt{\mu / \rho} / V$, and thus becomes unbounded as $\rho \rightarrow 0$ or $V \rightarrow 0^{+}$. Presumably, at low values of $\rho V^{2}$, the quasistatic growing crack solution of Rice and Nikolic (1985) is approached at moderate $r$. That solution gives a logarithmic (in $r$ ) infinity of $\gamma_{\alpha}^{\mathrm{p}}$, as $r \rightarrow 0$, for $\theta>\theta_{0}$ where $\theta_{0}$ is the angle of a velocity discontinuity in that case. It may be con- 
sidered valid in either limit $\rho \rightarrow 0$ or $V \rightarrow 0^{+}$. The finite strain of the dynamic solution, derived here. will presumably truncate the infinity of the quasistatic solution, but the details of the transition have not been worked out in this case. An analogous transition, but then only from weak to stronger singularity of strain, has been determined for the isotropic material (Freund and Douglas, 1982).

We now continue to examine other types of locally continuous solutions, within different angular sectors, for the governing equations. However. it does not appear that those local solutions can be combined to provide any acceptable overall solution for the near-tip field, as an alternative to that just presented.

\subsection{Elastic sectors}

These are the sectors of material that are currently responding elastically. If we substitute the form of velocity given with (2.12) into the equation of motion (2.4) and the constitutive equation (2.11) we obtain the basic equations in the following forms

$h_{\alpha_{\alpha}} \tau_{\alpha}^{\prime}=\rho V\left(A e_{1}-f^{\prime} h_{1}\right)$.

and

$-A e_{\alpha}+f^{\prime} h_{\alpha}=e_{2} V c_{\alpha \beta} \tau_{\beta}^{\prime}$

where $f^{\prime}=f^{\prime}(\theta)=\mathrm{d} f(\theta) / \mathrm{d} \theta$. Solving for $h_{\mathrm{c}} \tau_{\alpha}^{\prime}$ from (3.26) and substituting into (3.25) we can sove for $f^{\prime}(\theta)$ as

$$
\begin{array}{r}
f^{\prime}(\theta)=A\left[\left(\rho V^{2} e_{1} e_{2}+h_{\alpha} c_{\alpha \beta}^{-1} e_{\beta}\right)\right. \\
\left./\left(h_{\alpha} c_{\alpha \beta}^{-1} h_{\beta}-\rho V^{2} e_{2}^{2}\right)\right],
\end{array}
$$

and integrating (3.27) with respect to $\theta$ we can get the velocity field in the elastically responding sector as

$v=B-(A / 2) \ln \left[r^{2}\left(h_{\alpha} c_{\alpha \beta}^{-1} h_{\beta}-\rho V^{2} e_{2}^{2}\right)\right]$

where $B$ is a constant of integration. Note here that the denominator in (3.27) never vanishes if $e_{2} V$ is less than the elastic wave speed for the local $\theta$ direction. Using (3.27) in (3.26) we can also solve for the stress derivative as

$$
\begin{aligned}
\tau_{\alpha}^{\prime}(\theta)=\left(A / e_{2} V\right)\{ & {\left[c_{\alpha \beta}{ }^{1} h_{\beta}\left(\rho V^{2} e_{1} e_{2}+h_{\lambda} c_{\lambda \mu} e_{\mu \mu}\right)\right.} \\
& \left./\left(h_{\lambda} c_{\lambda \mu} h_{\mu}-\rho V^{2} e_{2}^{2}\right)\right] \\
& \left.-c_{\alpha \beta \beta}^{-1} e_{\beta}\right\} .
\end{aligned}
$$

If we recall the definitions of unit vectors $\boldsymbol{e}$ and $\boldsymbol{h}$ in terms of $\theta$, and consider the limit of stress derivatives, we note that $\tau_{1}^{\prime}$ behaves as $A(\sin \theta)^{1}$ near $\theta=0$ and $\pi$. i.e.. $\tau_{1}^{\prime}$ becomes unbounded at $\theta=0$ and $\pi$. Thus we conclude that an elastic sector can border $\theta=0$ or $\theta=\pi$ only if $A=0$ in (3.29). in which case we have constant velocity and stress field in this sector. Thus an elastic sector with variable stress and velocity fields cannot lie ahead of the crack and cannot border the crack surface.

3.3 Sectors at yield with plastic flow corresponding to a flat segment of the yield surface

The sectors of material that are stressed to yield can correspond either to the flat segment of the yield surface or to a vertex state. For the former case, considered here, the stresses satisfy

$m_{\alpha} \tau_{\alpha x}=\tau_{01}$

where $\tau_{0}$ is the yield stress and $m_{a x}$ is the normal to that flat. Note also that $\tau_{i x}^{\prime}=s_{\alpha} l^{\prime}$, from Fig. $1(\mathrm{~b})$, and $l^{\prime}=\mathrm{d} l / \mathrm{d} \theta$. If we take the scalar product of the constitutive equation (2.11) with $s_{\alpha x}$ (without the term in square brackets that corresponds to a vertex state), and also use (2.12) in the equation of motion (2.4), we obtain two equations for $l^{\prime}$ and $f^{\prime}$, in terms of the constant $A$, as

$$
\left[\begin{array}{cc}
h_{\alpha \alpha} s_{\alpha} & -\rho V e_{2} \\
-V s_{\alpha \alpha \beta} c_{\alpha \beta} s_{\beta} e_{2} & h_{\alpha} s_{\alpha}
\end{array}\right]\left\{\begin{array}{l}
l^{\prime} \\
f^{\prime}
\end{array}\right\}=A\left(\begin{array}{c}
\rho V e_{1} \\
s_{\alpha} e_{\alpha}
\end{array}\right) .
$$

The determinant of the matrix on the left-hand side of (3.31) is

$\operatorname{det}[\ldots]=\left(h_{\alpha} s_{\alpha}\right)^{2}-\rho V^{2} e_{2}^{2} s_{\alpha \gamma} c_{\alpha \beta} s_{\beta}$. 


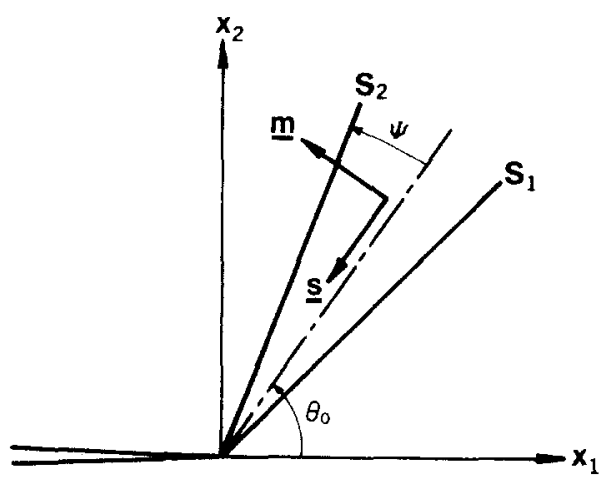

Fig. 3. Two lines along which determinant of eqn. (3.29) vanishes. The latter, $S_{2}$, is the same as the shock line in Fig. 2(b).

It is interesting to observe here that the condition for vanishing of this determinant, or at least its root with $h_{\alpha} s_{\alpha}>0$, is identical with the condition for a shock wave to exist at that value of $\theta$, given by (3.11). There are two lines along which determinant can vanish, Fig. 3. Since the determinant in (3.32) in the vicinity of either of these two lines behaves like $\left(\boldsymbol{\theta}-\boldsymbol{\theta}_{s_{i}}\right)$, where $\boldsymbol{\theta}_{s_{\text {, }}}$ denotes the position of either line, both $f(\theta) \rightarrow \infty$ and $l(\theta) \rightarrow$ $\infty$ on these lines if the constant $A$ is not equal to zero. Thus, a plastic sector of the type considered cannot contain either of these lines, even as a border, unless $A=0$. However, if $A=0$, then this sector has a constant velocity and stress field everywhere except along one of these two lines, on which $f^{\prime}(\theta)$ and $l^{\prime}(\theta)$ can take arbitrary values. If we allow them to be Dirac singular there, then these lines are sites of velocity and stress discontinuities and we recover the equations for shock discontinuities given earlier. From (3.31) we can solve for $l^{\prime}(\theta)$ and $f^{\prime}(\theta)$ as

$$
\begin{array}{r}
l^{\prime}=\rho V A\left\{\left(h_{\alpha} s_{\alpha} e_{1}+e_{\alpha} s_{\alpha} e_{2}\right)\right. \\
\left./\left[\left(h_{\mu} s_{\mu}\right)^{2}-\rho V^{2} e_{2}^{2} s_{\lambda} c_{\lambda \mu} s_{\mu}\right]\right\} \\
f^{\prime}=A\left\{\left(h_{\mu} s_{\mu} e_{\lambda} s_{\lambda}+\rho V^{2} e_{1} e_{2} s_{\lambda} c_{\lambda \mu} s_{\mu}\right)\right. \\
\left./\left[\left(h_{\alpha} s_{\alpha}\right)^{2}-\rho V^{2} e_{2}^{2} s_{\alpha} c_{\alpha \beta} s_{\beta}\right]\right\} .
\end{array}
$$

If we use $e_{\lambda}=-h_{\lambda}^{\prime}$ and $e_{1}=h_{2}=e_{2}^{\prime}$, and in- tegrate (3.34), we can solve for the velocity field of equation (2.12) for this sector as

$$
\begin{aligned}
v=\mathrm{constant}-(1 / 2) A \ln [ & r^{2} \mid\left(h_{\alpha} s_{\alpha}\right)^{2} \\
& \left.-\rho V^{2} e_{2}^{2} s_{\alpha} c_{\alpha \beta} s_{\beta} \mid\right] .
\end{aligned}
$$

To solve for $\Lambda$ we multiply the constitutive equation (2.11), with $m_{\alpha}$, assuming that $m$ is normalized so that $m_{\alpha} m_{\alpha}=1$, so that

$r A=m_{\alpha} r v,_{\alpha}-m_{\alpha} c_{\alpha \beta} e_{2} V \tau_{\beta}^{\prime}$.

Using (2.12) and the fact that $\tau_{\beta}^{\prime}=s_{\beta} l^{\prime},(3.36)$ becomes

$r \Lambda=-m_{\alpha} A e_{\alpha}+m_{\alpha} f^{\prime} h_{\alpha}-m_{\alpha} c_{\alpha \beta} e_{2} V s_{\beta} l^{\prime}$.

The relations

$m_{\alpha} h_{\alpha}=-e_{\alpha} s_{\alpha}$ and $m_{\alpha} e_{\alpha}=h_{\alpha} s_{\alpha}$

together with (3.33) and (3.34), used in (3.37), give

$$
\begin{aligned}
& r \Lambda=-h_{\alpha} s_{\alpha} A \\
& \quad-e_{\alpha} s_{\alpha} A\left\{\left[h_{\mu} s_{\mu} s_{\lambda} e_{\lambda}+\rho V^{2} m_{\alpha} c_{s s} e_{2}^{2}\right] / \operatorname{det}\right\} \\
& -\rho V^{2} A e_{1} e_{2}\left\{\left[h_{\alpha} s_{\alpha} c_{m s}+e_{\alpha} s_{\alpha} c_{s s}\right] / \operatorname{det}\right\},
\end{aligned}
$$

where det is the determinant of equation (3.32) and notations $c_{m s}$ and $c_{s s}$ have been explained earlier. Expression (3.39) in the elastically isotropic case reduces to

$$
\begin{array}{r}
r \Lambda=(-A / \mathrm{det})\left\{-h_{\mu} s_{\mu}\left[1-\left(\rho V^{2} / \mu\right) e_{2}^{2}\right]\right. \\
\left.-\left(\rho V^{2} / \mu\right) e_{1} e_{2} e_{\mu} s_{\mu}\right\} .
\end{array}
$$

where

$\operatorname{det}=\left(h_{\alpha} s_{\alpha}\right)^{2}-\left(\rho V^{2} / \mu\right) e_{2}^{2}$.

The requirement that $r \Lambda>0$ implies restrictions on the sign of $A$ in different regions.

\subsection{Sectors at yield corresponding to plastic flow at a vertex of the yield surface}

The stresses in this sector are constant, i.e., $\tau_{\alpha}^{\prime}=0$, and this used in the basic equations reduces them to

$0=-\rho V r v,{ }_{1}$ 
for the equation of motion, and

$r r_{\alpha}=r \Lambda m_{\alpha}+r \tilde{\Lambda} \tilde{m}_{\alpha}$

for the constitutive equation.

The consequence of (3.42), that $r v,_{1}=0$, used in (3.43) gives the relation

$r A m_{1}+r \tilde{\Lambda} \tilde{m}_{1}=0$.

Since both $\Lambda$ and $\tilde{\Lambda}$ are non-negative, (3.44) requires that $m_{1}$ and $\tilde{m}_{1}$ have to be of the opposite signs if both systems are to be plastically active. If $m_{1}$ and $\tilde{m}_{1}$ are of the same sign, then (3.44) can be satisfied only if $\Lambda=\tilde{\Lambda}=0$, so no plastically active sector is possible. This implies that sectors corresponding to vertices $B$ and $B^{\prime}$ on the $\tau_{1}$ axis of the yield surface cannot be plastically active vertex sectors, while sectors corresponding to the vertices like $A$ on the $\tau_{2}$ axis can; Fig. 1(b). Note that in the discontinuous solution proposed in Subsection 3.1, the angular sector corresponding to vertex $B$ was assumed to be non-deforming; the observation just made proves that it must be.

For continuity of velocity at the boundary between an active vertex sector and an elastic or active flat sector, the velocity at the boundary, $\theta=\theta_{h}$. has to be of the form

$\imath=A \ln r+f\left(\theta_{\mathrm{h}}\right)$

where $A$ and $f(\theta)$ pertain to the bordering sector. If we assume that $v$ is of the form

$v=-G(\theta) \ln r+F(\theta)$,

within the active vertex sector, where $F(\theta)$ and $G(\theta)$ are the unknown functions of angle, then we can calculate the derivative as

$r v,_{, \alpha}=\left[F^{\prime}(\theta)-G^{\prime}(\theta) \ln r\right] h_{\alpha}-G(\theta) e_{\alpha}$.

The vanishing of $r v_{1}$ that follows from (3.42), requires on the other hand that $G^{\prime}(\theta)=0$, leading to

$G(\theta)=$ constant $=A$,

and

$F^{\prime}(\theta)=A\left(e_{1} / h_{1}\right)=A[\cos \theta /(-\sin \theta)]$. i.e.,

$F(\theta)=$ constant $-A \ln |\sin \theta|$.

Thus, under the above assumption (3.46), about the mathematical structure of the velocity function, it is necessary that

$$
\begin{aligned}
v & =\mathrm{constan} t-A \ln (r|\sin \theta|) \\
& =\mathrm{constan} \mathrm{t}-A \ln \left|x_{2}\right| .
\end{aligned}
$$

Therefore, a plastically active sector can extend to $x_{2}=0$, (i.e., to $\theta=0, \pi$ ) only if $A=0$, but for that case it is not really active since both $r \Lambda \rightarrow 0$ and $r \tilde{\Lambda} \rightarrow 0$. Here we note that in the discontinuous solution proposed earlier the sector corresponding to vertex $A$ was assumed to be non-deforming and, within the assumption of (3.46), the last result shows that it must be so.

A strengthened argument, replacing the assumption (3.46), may be made as follows when the crack advances under steady state conditions so that $\dot{v}=-V v, 1$. Then, since $\tau_{\alpha}=$ constant in a region corresponding to a vertex, (2.1) with zero body force implies $v_{1_{1}}=0$ and $v=h\left(x_{2}\right)$ in that region. If we interpret (3.45) as being valid up to terms of $o\left(r^{\circ}\right)$ near $r=0$, then for continuity of $v$ at the sector border $\theta=\theta_{\mathrm{h}}$ we have (setting $x_{2}=$ $r \sin \theta_{\mathrm{b}}$ at the border)

$$
\begin{aligned}
v & =h\left(x_{2}\right) \\
& =A \ln \left|x_{2}\right|+f\left(\theta_{\mathrm{b}}\right)-A \ln \left|\sin \theta_{\mathrm{h}}\right|+o\left(x_{2}^{0}\right)
\end{aligned}
$$

as $x_{2} \rightarrow 0$. This reproduces (3.51) and has the same consequences.

\section{Assembly of sectors and lack of alternate solu- tions to that with elastic-plastic shock}

In deriving the solutions of the governing equations for the stress and strain fields around the tip of a dynamically growing crack we have encountered some restrictions that apply to different types of sectors. These enable us to rule out different possiblities of solutions other than the discontinuous solution already given in subsection 3.1 and Fig. 2(b). 


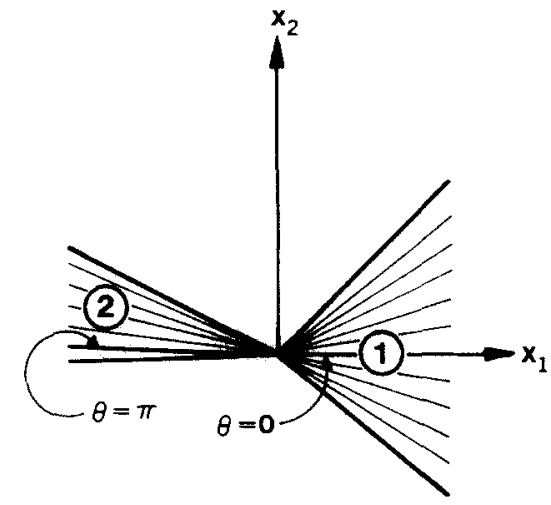

(a)

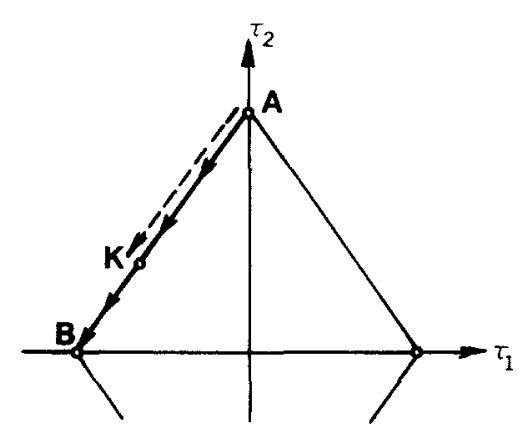

(b)

Fig. 4. Tentative sectors around the crack tip: sector 1 bordering on $\theta=0$ and sector 2 bordering on $\theta=\pi$. (b) The tentative jump from vertex $A$ to a general state $K$ along the flat (it is shown in the text that $K$ must coincide with vertex $B$ ).

The sector ahead of the crack, bordering on $\theta=0$, (sector 1, Fig. 4(a)) cannot be a plastically active vertex sector, nor can it be a deforming elastic sector. Either hypothesis as to a sector type will lead to the requirement that $A=0$, in which case the sector is inactive, constant stress type (eqns. (3.48) and 3.29)). Thus we conclude that this sector may be either of the type constant stress but inactive, or of type plastically active corresponding to a single flat segment of the yield surface.

The sector bordering the crack wall, at $\theta=\pi$, may not be a plastically active vertex sector, nor may it be a deforming elastic sector (sector 2, Fig 4(a)). This too must be either constant stress but inactive, or plastically active corresponding to a single flat segment of the yield surface.

Now we consider the possiblity that the sector ahead of the crack, 1 , is plastically active corresponding to a single flat. Then the velocity field is given by

$v=$ constant

$$
-(1 / 2) A \ln \left[r^{2}\left|\left(h_{\alpha} s_{\alpha}\right)^{2}-\rho V^{2} e_{2}^{2} c_{s s}\right|\right] .
$$

The symmetry requires that $v=0$ on $\theta=0$, and this condition can be met only if $A=0$, which, in turn, means that the sector is not plastically active, since $r \Lambda \rightarrow 0$ if $A=0$. Thus one concludes that the sector in front of the crack must be of constant stress, plastically inactive type (in the sense that $r \Lambda \rightarrow 0$ ). The stress state is at a vertex ( $A$ of
Fig. 1(b), assuming $\tau_{2}>0$ ahead of the crack) since a constant stress state that is not on the yield locus has no possibility of varying and cannot meet crack surface conditions.

Let us now assume that the constant state corresponding to a vertex prevails until a jump is allowed. As established in Section 3.1 (equation (3.12) and discussion following after it), the first permissible jump is at

$h_{\alpha} s_{\alpha}=V e_{2} \sqrt{\rho c_{s . s}}$.

We have to check how far the jump can go (Fig. 4(b)). It cannot go only part-way to the other vertex, because then the adjoining sector, if plastic, would be plastically active corresponding to single flat type ( $K$, Fig. $4(b)$ ). As established in Section 3.3 , such a sector can border a line meeting the shock condition only when $A=0$, in which case it is of constant stress, plastically inactive type. Thus if we assume no elastic unloading sector, the sector after the shock must be of constant stress, plastically inactive type with stress at $K$. Since no further jump is possible for $\theta>\theta_{0}+\psi$, $K$ must be chosen so that it meets the crack boundary conditions. Hence $K$ must correspond to vertex $B$, Fig. 4(b), and this reproduces the discontinuous solution discussed earlier.

The conclusion that can be drawn thus far is that if all sectors around the crack are at yield (i.e., if there is no elastically deforming unloaded 
sector) the only solution is the one with two constant stress, plastically inactive sectors that are joined by the shock. Fig. 2(b). This type of solution is consistent with the conclusions of Drugan and Shen (1987) and satisfies the crack surface boundary condition.

We now consider why elastic sectors cannot, in fact, intervene in the constant stress, plastically inactive sectors just discussed. Consider the sector beginning at $\theta=0$, corresponding to vertex $A$ of Fig. 1(b). We have observed already that the prelogarithmic velocity constant $A$ of (2.12) is zero in this sector. Hence, for velocity continuity (which must be met, according to our shock analysis, for all $\theta$ except for $\left.\theta=\theta_{0}+\psi\right), A=0$ must be met also in any elastic (or plastically active along a single flat) sector which begins to emerge prior to $\theta=\theta_{0}+\psi$. But the condition $A=0$ converts those to constant stress sectors and, since stress too must be continuous, except possibly at $\theta=\theta_{0}+\psi$, the constant stress sector beginning at $\theta=0$ must extend as a constant stress sector to $\theta=\theta_{0}+\psi$.

If there is no discontinuity at $\theta=\theta_{0}+\psi$, the constant stress sector beginning at $\theta=0$ would have to extend, by similar reasoning, to $\theta=\pi$. This would violate the crack surface boundary condition, and thus a discontinuity is required. By the analysis of the discontinuity in Section 3.1, e.g., eqn. (3.22), the jump $v^{-}-v^{+}$has no $r$ variation, and thus since $A$ of (2.12) vanishes for $\theta=\theta_{0}$ $+\psi^{-}$, so also must $A$ vanish for $\theta=\theta_{0}+\psi^{+}$which we may (tentatively) assume to correspond to some general state $K$ as in Fig. 4(b). The reasoning now is the same as in the previous paragraph. Any elastic sector which was assumed to emerge would (by the requirement of velocity continuity if emerging at $\theta>\theta_{0}+\psi$, or by the shock analysis if at $\theta=\theta_{0}+\psi^{+}$) have $A=0$, and thus would be no more than a constant stress sector carrying the same stress as at $K$. Hence the shock must be followed by a constant stress sector at $K$, for all $\theta>\theta_{10}+\psi^{+}$, and the boundary condition on the crack face again requires that $K$ coincides with point $B$.

Thus we conclude that the discontinuous neartip solution, with an elastic-plastic shock between non-deforming constant stress sectors, is the only possible near-tip field giving $\tau_{2}>0$ ahead of the crack when $\rho V^{2} \neq 0$.

It will be of interest to learn if similar features to those discussed here apply to dynamic growth of elastic-plastic tensile cracks in ductile crystals. The case of quasistatic growth (i.e.. limits $\rho \rightarrow 0$ or $V \rightarrow 0^{+}$) has been analyzed recently for the plane strain tensile crack in ideally plastic crystals (Rice. 1987), and leads to logarithmic-in-r strain singularities at the tip, just as in the corresponding anti-plane solution (Rice and Nikolic. 1985). If the strain singularities for the tensile case are truncated by inertial effects to finite crack tip strains, as demonstrated here for anti-plane strain, such could be important to understanding the resistance of ductile materials to rapid dynamic crack growth.

\section{Acknowledgement}

This research was supported by the Office of Naval Research under contract N00014-85-K-0045 with Harvard University.

\section{References}

Achenbach, J.D. and V. Dunayevsky (1981), Fields near a rapidly propagating crack-tip in an elastic-perfectly plastic material, J. Mech. Phys. Solids 29 (4), 283.

Drugan, W.J. and J.R. Rice (1984), Restrictions on quasi-statically moving surfaces of strong dicontinuity in elastic-plastic solids, in: G.J. Dvorak and R.T. Shield, eds., Mechunics of Material Behavior (the D.C. Drucker anniversary volume), eds., Elsevier, Amsterdam, p. 59

Drugan, W.J. and Y. Shen (1987). Restrictions on dynamically propagating surfaces of strong discontinuity in elastic-plastic solids, J. Mech. Phys. Solids 35 (6), 771

Freund L.B. and A.S. Douglas (1982), The influence of inertia on elastic-plastic anti-plane shear crack growth, $J$. Mech. Phys. Solids $30(1 / 2), 54$.

Rice, J.R. and R. Nikolic (1985), Anti-plane shear cracks in ideally plastic crystals. J. Mech. Phys. Solids 33 (6), 595.

Rice, J.R. (1987), Tensile crack tip fields in elastic-ideally plastic crystals, Mech. Mater. 6 (4), 317.

Slepyan, L.I. (1976), Crack dynamics in elastic plastic body, Izvestiva Akademii Nauk SSSR, Mekhanika Tverdogo Tela II (2) 144 